\title{
Metastasectomy as optimal treatment for late relapsing solitary brain metastasis from testicular germ cell tumor: a case report
}

Keitaro lida, Taku Naiki ${ }^{*}$, Noriyasu Kawai, Ryosuke Ando, Toshiki Etani, Keiichi Tozawa and Kenjiro Kohri

\begin{abstract}
Background: Management of late relapse of a testicular germ cell tumor is difficult because few cases have been reported and the tumors are intractable to chemotherapy. Here we present a case with a single brain metastasis from late relapse of a testicular germ cell tumor. This is the first report of a brain metastasis that was treated successfully only by surgery.

Case presentation: A 19-year-old Japanese man presented with breathing difficulties and left testis enlargement and he was diagnosed with a yolk sac tumor following a left orchiectomy. At the time of diagnosis, multiple lung metastases were apparent on computed tomography, and serum alpha-fetoprotein level was elevated to $10,245 \mathrm{ng} / \mathrm{ml}$. The patient received three postoperative courses of bleomycin, etoposide and cisplatin and etoposide and cisplatin respectively and a complete response was obtained. Four years after surgery, the patient was admitted to the hospital due to a sudden seizure. High alpha-fetoprotein levels $(539 \mathrm{ng} / \mathrm{ml})$ were evident and magnetic resonance imaging suggested a $45-\mathrm{mm}$ single brain tumor in the right parietal lobe, for which surgery was performed. The pathological diagnosis was yolk sac tumor. The alpha-fetoprotein level remained normal at 2 months after operation. There was no recurrence 24 months post-operation.
\end{abstract}

Conclusion: Chemoresistance and late neurotoxicity are concerns in treating brain metastasis with chemotherapy or cerebral radiotherapy. Surgery is believed to be the optimal treatment choice if the size of the brain metastasis is larger than 35-mm and the late relapse area is surgically accessible.

Keywords: Late relapse, Testicular germ cell tumor, Yolk sac tumor, Brain metastasis, Surgery

\section{Background}

Late relapses (LRs) of a testicular germ cell tumors (GCT) is defined as a recurrence after complete response to treatment with a subsequent disease-free interval of at least of 2 years [1]. LRs of testicular GCT are rare, with an incidence rate of 3- 4\% [2]. The most common sites of LR are the retroperitoneum (47\%), lungs (25\%) and mediastinum (10\%) [1]. LRs also appear in cervical nodes (8\%) and the liver (6\%) [1]. To date, only a few cases of brain metastases of LR of testicular GCTs have been reported. Hence, it is quite difficult to decide on the choice of treatment.

Herein, our experience in a case with a single brain metastasis from a LR of a testicular GCT is described.

\footnotetext{
* Correspondence: rx-nike@hotmail.co.jp

Department of Nephro-urology, Nagoya City University Graduate School of Medical Sciences, Kawasumi 1, Mizuho-cho, Mizuho-ku, 467-8601 Nagoya, Japan
}

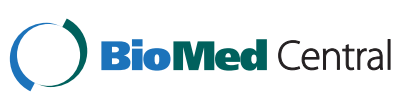

The patient was successfully treated with surgery alone. This paper describes the characteristics and treatment of LRs of testicular GCTs including brain metastases.

\section{Case presentation}

A 19-year-old Japanese man consulted a family physician due to breathing difficulties. The left testis was enlarged to $15 \mathrm{~cm}$ in diameter, and multiple lung shadows were observed on a chest X-ray. The patient was then referred to our institution due to suspected lung metastases of testicular cancer. Left orchiectomy was performed and he was diagnosed with a yolk sac tumor (YST). At the time of diagnosis, multiple lung metastases were apparent on computed tomography (CT). Postorchiectomy serum levels of tumor markers were examined, with an elevated alphafetoprotein (AFP) level of 10,245 $\mathrm{ng} / \mathrm{ml}$, but otherwise normal with lactate dehydrogenase (LDH) level of $151 \mathrm{U} / \mathrm{l}$ and 
human chorionic gonadotrophin (HCG) level of 0.4 IU/ L. Elevated AFP level indicated that he fulfilled the criteria of poor prognosis according to the International Germ Cell Consensus Classification [3]. The patient received three postoperative courses of bleomycin, etoposide and cisplatin (BEP) and etoposide and cisplatin (EP) respectively and a complete response was obtained. There was no recurrence post-operatively during follow-ups at 3-6 months intervals, according to serum marker levels and CT imaging from the chest to pelvis. Four years after surgery, the patient was transferred to the emergency department due to a sudden seizure. High AFP levels (539 $\mathrm{ng} / \mathrm{ml}$ ) were evident, although other markers were normal. Magnetic resonance imaging (MRI) suggested a 45-mm single brain tumor in the right parietal lobe, and no other tumor was evident, including the contralateral testis (Figure 1). Surgery was performed on the single brain metastasis. The pathological diagnosis was YST (Figure 2). The AFP level remained normal at 2 months after surgery. The patient has not had neurological disability. Moreover, there was no recurrence 24 months after surgery.

\section{Conclusions}

LR can be due to a second primary tumor (either a metastasis from a second testicular neoplasm or an extragonadal primary tumor), a delayed transformation of a

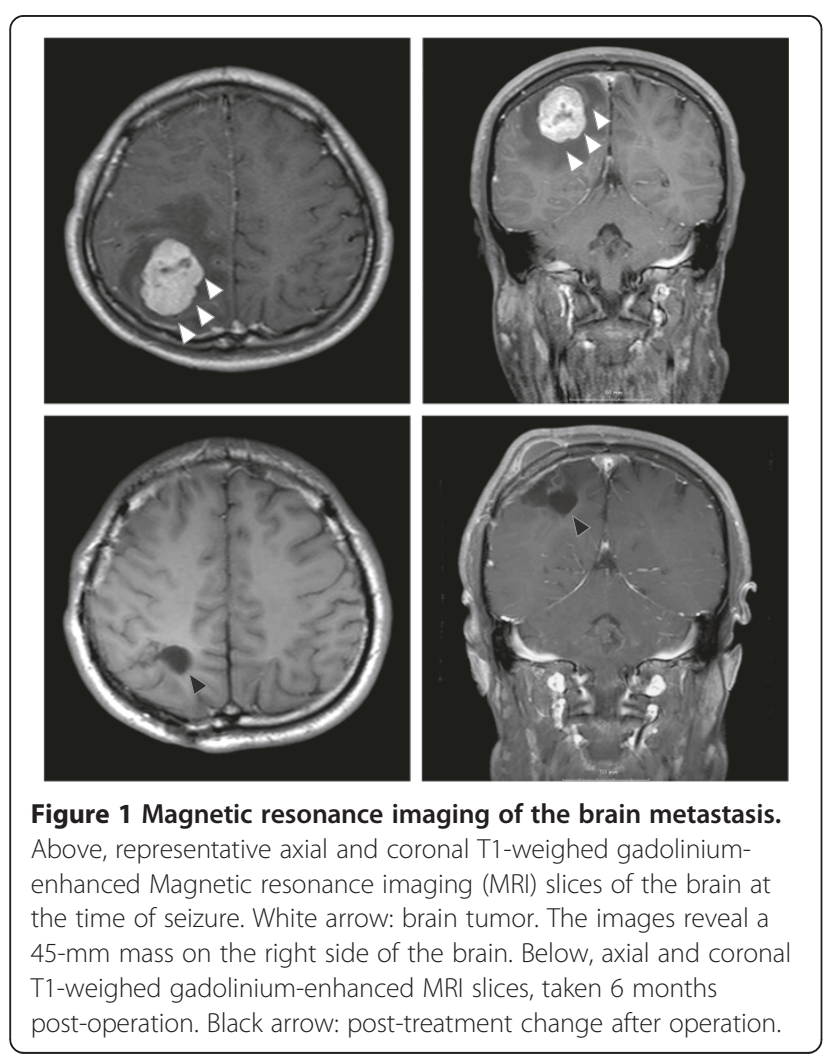

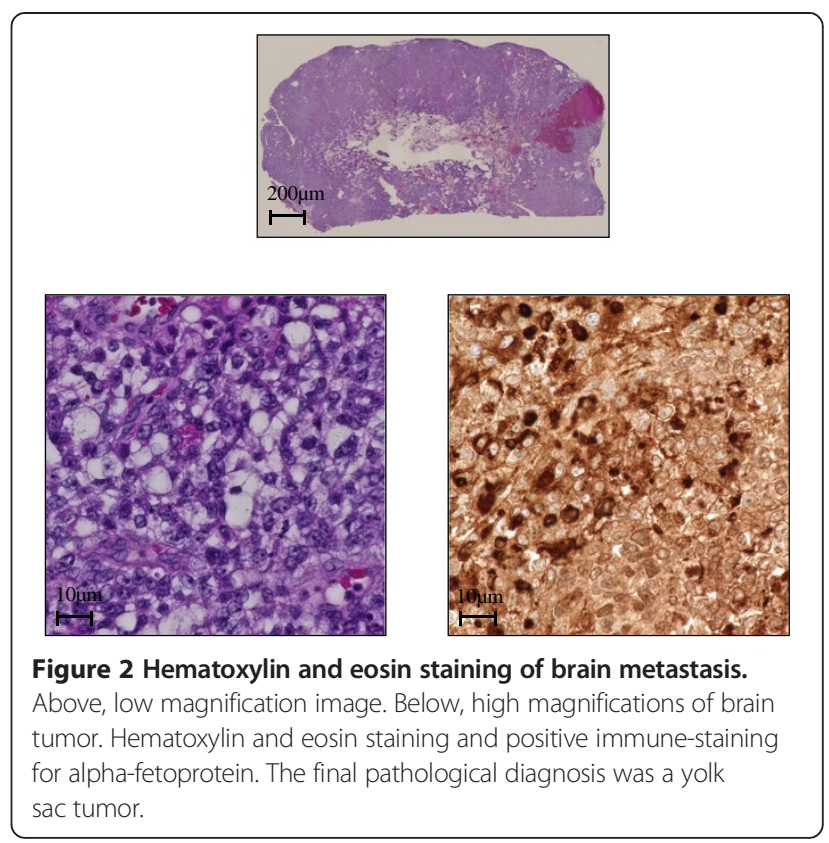

metastatic teratoma into a malignant neoplasm, or persistence of a microscopic viable cancer with slowgrowing tendencies [4].

Various histologies have been reported for LR: GCT (66\%), pure teratoma (17-22\%), sarcoma (6\%), and adenocarcinoma $(6 \%)[1,2,5]$. Sixty percent of patients present with elements of teratoma [5]. Of those patients with LR of GCT, $44.6 \%$ are diagnosed with YSTs, which comprise the most common GCT subtype [2]. A report by Michael et al. included 91 patients with LR of GCT, of whom 43 had YSTs [5]. Of those 43 patients with LR of GCT, only 12 patients were diagnosed with YST as the primary neoplasm. Furthermore, the disease-free rates for patients with LR during long-term follow-up were $74 \%$ for those with LR consisting only of teratoma versus, $37 \%$ for those with LR consisting of YST.

Testicular GCT responds well to chemotherapy, even with brain metastases, but chemotherapy for LR has been unsuccessful in many patients [5]. According to a study by Baniel, 17 of 65 (26\%) patients who received chemotherapy as treatment for LRs of testicular GCTs experienced complete responses. However, only 2 (3\%) patients who received chemotherapy alone were continuously diseasefree. Of note, these 2 patients had not previously received cisplatin-based chemotherapy, suggesting that LR is associated with chemoresistance [1,5]. While chemotherapy might be effective for chemotherapy-naive patients, it does not work for most patients with LR if they previously received chemotherapy to treat the primary tumor. Consequently, a surgical approach is the most appropriate treatment choice. George et al. reported that 49 of 83 patients (59\%) presented with no evidence of disease after surgery for LR [6]. Twenty-one of the 49 patients (43\%) 
who responded successfully to the primary surgery remained continuously disease-free, while 28 of the 49 patients (57\%) experienced one or more subsequent relapses.

The 5-year cause-specific survival for all cases of brain metastasis from testicular GCT ranges from $35.9 \%$ to $-53 \%$ [7-9]. The 5-year cause- specific survival for cases of brain metastasis at initial diagnosis was $42.9 \%$. In contrast, the 5year cause-specific survival for cases of brain metastasis after or during cisplatin-based chemotherapy was even worse, $12 \%$ to $-29.6 \%$, indicating that brain metastases in the latter group were chemotherapy-resistant [7-9]. In such cases, cerebral radiotherapy or surgery might provide important benefits.

Cerebral radiotherapy has been demonstrated as a treatment for brain metastases of testicular GCT, as these tumors possess a certain level of radiosensitivity. Stereotactic radiotherapy (SRT) techniques, such as GammaKnife or CyberKnife have been used to treat brain tumors. In general, for brain metastasis, the indication for SRT includes tumors with a median diameter less than $3.5 \mathrm{~cm}$ and up to 3 small brain metastases. Metastases in the eloquent cortex, basal ganglia, thalamus, and even the brainstem, a location considered to be surgically inaccessible, can be treated with a relatively low risk [10]. In clinical practice, surgery should be considered for any patient with a single brain metastasis in an accessible location, especially if the tumor is large, the mass effect is significant, and/or obstructive hydrocephalus is present [11]. In our case, SRT was not chosen because the tumor size was $4.5 \mathrm{~cm}$ and it was accessible by surgery.

In whole-brain radiation therapy (WBRT) for brain metastases, a total of 30 to $50 \mathrm{~Gy}$, divided in 10-20 fractions is applied, and with a single dose is $1.5-3$ Gy $[8,9]$. WBRT alone could be a treatment option for patients with multiple brain metastases or poor performance status patients [11]. WBRT is sometimes supplemented with surgery or SRT to treat micrometastases [8,9]; however, there is no definitive evidence of overall survival imporvement if WBRT is performed after surgery, instead of SRT. Additionally, the risk of late neurotoxicity after WBRT in longsurviving patients is not negligible. Up to $11 \%$ of patients who underwent WBRT experienced neurotoxicity symptoms, including memory loss that progressed to dementia, frontal gait disorders, and urinary incontinence [11].

In our case, the LR of YST, discovered 4 years after operation was localized in a surgically accessible area of the patient's brain. SRT was not chosen but a metastasectomy was performed in consideration of the tumor size and location. Because tumor resection was successful, chemotherapy or cerebral radiotherapy was not applied due to concerns about chemoresistance and late neurotoxity. There has been no recurrence 24 months post-operation. Thus, surgery is considered an optimal treatment choice of the LR area if surgically accessible.

\section{Consent}

Written informed consent was obtained from the patient for publication of this Case report and any accompanying images. A copy of the written consent is available for review by the Editor of this journal.

\section{Abbreviations}

LR: Late relapse; GCT: Germ cell tumor; YST: Yolk sac tumor; CT: Computed tomography; AFP: Alpha-fetoprotein; LDH: Lactate dehydrogenase; HCG: Human chorionic gonadotrophin; BEP: Bleomycin, etoposide, and cisplatin; EP: Etoposide and cisplatin; MRI: Magnetic resonance imaging; SRT: Stereotactic radiotherapy; WBRT: Whole-brain radiation therapy.

\section{Competing interests}

The authors declare that they have no competing interests.

\section{Authors' contributions}

All the authors have read and approved the manuscript and agree with its submission to your journal. Details regarding authorship, conflicts of interest, and ethical approval are given in the accompanying Author Submission Requirement Form. Each author participated sufficiently in the work to take public responsibility for appropriate portions of the content. KI made diagnosis of this case and drafting of the manuscript. TN made critical revision of the manuscript. NK, RA, TE, and KT carried out the acquisition of data, and coordination and helped to draft the manuscript. KK made supervision of this manuscript. All authors read and approved the final manuscript.

Received: 20 July 2014 Accepted: 18 November 2014

Published: 2 December 2014

\section{References}

1. Baniel J, Foster RS, Gonin R, Messemer JE, Donohue JP, Einhorn LH: Late relapse of testicular cancer. Clin Oncol 1995, 13:1170-1176.

2. Sato S, Tanaka T, Takahashi A, Sasai M, Kitamura H, Masumori N, Tsukamoto $\mathrm{T}$ : Late recurrence and second primary malignancy among 139 patients with germ cell tumors: long-term outcome of the disease in a single-center experience. Jpn J Clin Oncol 2010, 40:157-162.

3. The International Germ Cell Cancer Collaborative Group: International germ cell concensus classification: A prognostic factor-based staging system for metastatic germ cell cancers. J Clin Oncol 1997, 15:594-603.

4. Lipphardt ME, Albers P: Late relapse of testicular cancer. World J Urol 2004, 22:47-54.

5. Michael H, Lucia J, Foster RS, Ulbright TM: The pathology of late recurrence of testicular germ cell tumors. Am J Surg Pathol 2000, 24:257-273.

6. George DW, Foster RS, Hromas RA, Robertson KA, Vance GH, Ulbright TM, Gobbett TA, Heiber DJ, Heerema NA, Ramsey HC, Thruston VC, Jung SH, Shen J, Finch DE, Kelley MR, Einhorn LH: Update on late relapse of germ cell tumor: a clinical and molecular analysis. J Clin Oncol 2003, 21:113-122.

7. Nonomura N, Nagahara A, Oka D, Mukai M, Nakayama M, Nishimura K, Kakimoto K, Nakamura T, Usami M, Okuyama A, Miki T: Brain metastases from testicular germ cell tumors: a retrospective analysis. Int J Urol 2009, 16:887-893.

8. Fosså SD, Bokemeyer C, Gerl A, Culine S, Jones WG, Mead GM, Germa-Luch JR, Pont J, Schmoll HJ, Tjulandin S: Treatment outcome of patients with brain metastases from malignant germ cell tumors. Cancer 1999, 85:988-997.

9. Salvati M, Piccirilli M, Raco A, Santoro A, Frati R, Lenzi J, Lanzetta G, Agrillo A, Frati $A$ : Brain metastasis from non-seminomatous germ cell tumors of the testis: indications for aggressive treatment. Neurosurg Rev 2006, 29:130-137.

10. Robert C, Aman S, David S, Patrick W: Cancer and the Nervous System. In Bradley's Neurology in Clinical Practice. 6th edition. Edited by Robert BD, Gerald MF, Joseph J, John CM. ; 2012:1184-1188.

11. Soffietti R, Rudā R, Mutani R: Management of brain metastases. J Neurol 2002, 249:1357-1369.

doi:10.1186/1756-0500-7-865

Cite this article as: lida et al.: Metastasectomy as optimal treatment for late relapsing solitary brain metastasis from testicular germ cell tumor: a case report. BMC Research Notes 2014 7:865. 